\title{
Borrelia Ocular Infection: A Case Report and a Systematic Review of Published Cases
}

\author{
Björn E. Lindström ${ }^{a, b}$ Barbro H. Skogman ${ }^{b, c}$ Annika K. Lindström ${ }^{d}$ \\ Leif Tallstedt ${ }^{\mathrm{e}}$ Kenneth Nilsson ${ }^{\mathrm{f}, \mathrm{g}}$ \\ aDepartment of Ophthalmology, Falu Hospital, Falun, Sweden; ${ }^{b}$ Center for Clinical Research, Dalarna, Uppsala \\ University, Uppsala, Sweden; 'Faculty of Medical and Health Sciences, Örebro University, Örebro, Sweden; \\ ${ }^{d}$ Department of Women's and Children's Health, Uppsala University, Uppsala, Sweden; 'Department of \\ Ophthalmology, St Erik's Eye Hospital, Stockholm, Sweden; 'Department of Medical Sciences, Section of Clinical \\ Microbiology, Uppsala University, Uppsala, Sweden; 9Department of Medical Sciences, Section of Infectious \\ Diseases, Uppsala University, Uppsala, Sweden
}

\section{Keywords}

Borrelia spirochetes · Inflammation/uveitis · Ocular tissue · Polymerase chain reaction · Microscopy

\begin{abstract}
Introduction: Lyme borreliosis can cause many diverse manifestations, also ocular disease where the diagnosis of ocular borreliosis is challenging. The primary aim was to report on the evidence of Borrelia spirochetes in the ocular tissue in presumed ocular borreliosis. Methods: A systematic review of pathological eye conditions was performed where Borrelia has been suspected in relevant ocular tissue, together with a case report of diagnosed uveitis with polymerase chain reaction (PCR)-confirmed Borrelia afzelii in the vitreous. The evidence for clinical and laboratory diagnosis was evaluated systematically. As a secondary aim, the treatment of ocular Borrelia infection was also evaluated for confirmed cases. $\boldsymbol{R e}$ sults: Thirteen includable studies were found, and after the removal of case duplicates, eleven unique cases were extracted. Apart from the present case report, 4 other cases reported strong evidence for the detection of $B$. spirochetes in ocular tissue. Four cases presented reasonable evidence
\end{abstract}

for assumed detected Borrelia, while three additional cases showed only weak diagnostic credibility that Borrelia was detected. Conclusion: This systematic review, including all reported cases and our case report, supports evidence of ocular infection of Borrelia species. Furthermore, in case of suspicion of infection and seronegativity, it is justified to look for Borrelia in eye tissue samples. In addition, microscopy without using PCR is not sufficient to confirm the diagnosis of borreliosis on ocular tissue. In the articles studied, there was no unambiguous recommendation of treatment.

\footnotetext{
(C) 2022 The Author(s).

Published by S. Karger AG, Basel
}

\section{Introduction}

Lyme borreliosis, or Lyme disease, caused by Borrelia spirochetes, usually transmitted to humans by ticks in the temperate climates, can cause many diverse manifestations, especially in the early stages, before a serological response has developed, establishing that the diagnosis may be complex. Not least, the diagnosis of ocular borreliosis can be challenging. The diagnostic possibilities
C 2022 The Author(s).

Published by S. Karger AG, Basel

This is an Open Access article licensed under the Creative Commons Attribution-NonCommercial-4.0 International License (CC BY-NC) (http://www.karger.com/Services/OpenAccessLicense), applicable to the online version of the article only. Usage and distribution for commercial purposes requires written permission.
Correspondence to:

Kenneth Nilsson, kenneth.nilsson@medsci.uu.se 
provided by serology and clinical findings are limited, and biopsies of eye tissue are seldom without risk. Antibiotic treatment attempts of presumed eye disease caused by Borrelia species (spp.) are often arduous and not always of clear benefit. In the differential diagnosis of uveitis, the awareness of these difficulties can lead to a lowered interest in confirming Borrelia as a possible genesis.

In the French guidelines of 2019 for Lyme disease, ophthalmological manifestations were given evidence grade B by Gocko et al. [1]. This was motivated by most reports of ocular borreliosis being based only on serological findings, which cannot guarantee Borrelia as the cause of the eye condition. The French guidelines referred only to one case of $B$. spirochetes detected in eye tissue reported by Dietrich et al. [2], and two reports of ophthalmological manifestations (uveitis) concomitant to Lyme neuroborreliosis, confirmed by cerebrospinal fluid (CSF) analysis. The French authors concluded that the evidence of ocular Borrelia infection is weak. In our current article, we have performed a systematic review of the literature and methodology for a series of those already published cases to assess the evidence that support an ocular infection of Borrelia spp. We also present our case of uveitis with polymerase chain reaction (PCR)-verified Borrelia afzelii in the vitreous, which was the incentive for this review.

\section{Case Presentation}

The patient was a 51-year-old man with mild diet-treated diabetes, otherwise healthy and fit, spending a lot of time outdoors. In September 2016, he was examined because of a one-day history of floaters in the left eye (LE). His best-corrected visual acuity (BCVA) was in decimal Snellen 1.0 in the right eye (RE) and 0.7 in the LE. He was diagnosed as having vitreous detachment. At this time, he had a possible frozen shoulder and itchy skin rash on the abdomen. Two months later, he had metamorphopsia in the LE, and another month later, BCVA was reduced to 0.4. At the examination, both eyes were white with no inflammatory signs in the RE. In the LE, there were small keratic precipitates, $2+$ cells in the anterior chamber, a lot of cells and cell aggregates in the vitreous, and a macular epiretinal membrane but no signs of inflammatory retinal changes. He was given $30 \mathrm{mg}$ of oral prednisolone with a good effect on the vitreous cells, and BCVA was 1.0 after one week. C-reactive protein, hemoglobin, platelet count test, white blood cell count, serum ionized calcium, and computed tomography of the chest were normal. Prednisolone was withdrawn and an intravitreal implant of $700 \mathrm{mg}$ dexamethasone $\left(\right.$ Ozurdex $\left.^{\circledR}\right)$ was given, in spite of the fact that the patient was not assessed for associated diseases. Three months later, in March 2017, his BCVA decreased to 0.7 in the LE, and there were again cells in the anterior chamber and vitreous, but this time some retinal hemorrhages and multiple white dots in the inferior part of the retina were observed. Further investigation showed normal values of s-angiotensin-converting enzyme, syphilis serology, serum antineutrophil cytoplasmic antibodies, plasma (P)-immunoglobulin (Ig) A, P-IgG, P-IgM, P-orosomucoid, P-albumin, and P-haptoglobin. Borrelia serology was IgG-positive $(\geq 200 \mathrm{RU} / \mathrm{mL})$, IgM $21 \mathrm{RU} / \mathrm{mL}(<16 \mathrm{RU} / \mathrm{mL}=$ no detection; $16-21 \mathrm{RU} / \mathrm{mL}=$ borderline; and $>21 \mathrm{RU} / \mathrm{mL}=$ detection). He had no history of a tick bite (TB) or erythema migrans (EM). He was given another Ozurdex ${ }^{\circledR}$ and also a 10-day course of oral doxycycline $200 \mathrm{mg}$ per day.

During the following year, another three Ozurdex ${ }^{\circledR}$ injections were given, each with an effect of about three months. In March 2018, a new evaluation was performed with the same level of Borrelia antibodies as a year earlier and other blood tests were normal. Fluorescein angiography of the retina and magnetic resonance tomography of the brain were normal. Another identical course of oral doxycycline was given.

In May, a cataract extraction was performed successfully, and in September 2018, a vitrectomy was performed mainly for diagnostic purposes. The vitreous was sent for laboratory diagnostics. Flow cytometry of the vitreous cells showed reactive $\mathrm{T}$ lymphocytes with a CD4/CD8 ratio of 1.4 and a normal expression of CD5. There were no B cells or granulocytes and no suspicion of lymphoma. With PCR, there was no detection of adenovirus, herpes simplex virus 1 , herpes simplex virus 2 , varicella-zoster, or cytomegalovirus DNA. However, 16s rRNA PCR was positive, where the following sequence analysis showed complete homology with B. afzelii. The PCR analysis was performed as part of routine diagnostics at the Department of Clinical Microbiology, Karolinska University Hospital, which is under the regulation of the Swedish Board for Accreditation and Conformity Assessment, Swedac. At the time of vitrectomy, a new Borrelia serology showed positive titers for IgG (180 RU/mL) and IgM (24 RU/mL).

A two-week course of intravenous ceftriaxone $2 \mathrm{~g}$ per day was given. At follow-up, there was an epiretinal membrane with discrete edema which was later resolved with a course of topical corticosteroids and nepafenac. There have been occasional cells in the anterior chamber. The topical treatment was postponed in August 2019. In January 2020, the eye was quiet without cells or macular edema, and the VA was 1.0. In June 2020, the eye had only a few cells in the anterior chamber and the vitreous, BCVA was 1.0 LE, and a parafoveal intraretinal cyst was noticed. A low dose of topical steroids was reinstituted which dried up macula and has kept the vitreous and anterior chamber free, or nearly free of inflammatory cells, and the last visit was on the twentieth of July 2021. There were never any signs of uveitis on the RE.

\section{Methods}

\section{Study Selection and Used Criteria}

This systematic review followed the Preferred Reporting Items for Systematic Reviews and Meta-Analyses Protocols guidelines [3]. Review protocols were developed accordingly, both for the search and the review process (online suppl. material 1, 2; see www. karger.com/doi/10.1159/000521307 for all online suppl. material). The systematic review at PubMed was conducted in January 2019 for eligible articles that fulfilled all 3 of our chosen inclusion criteria: (A) case/cases with pathology in the tissue of the eyeball itself, the ocular adnexa, the extraocular muscles, and the optic nerve 


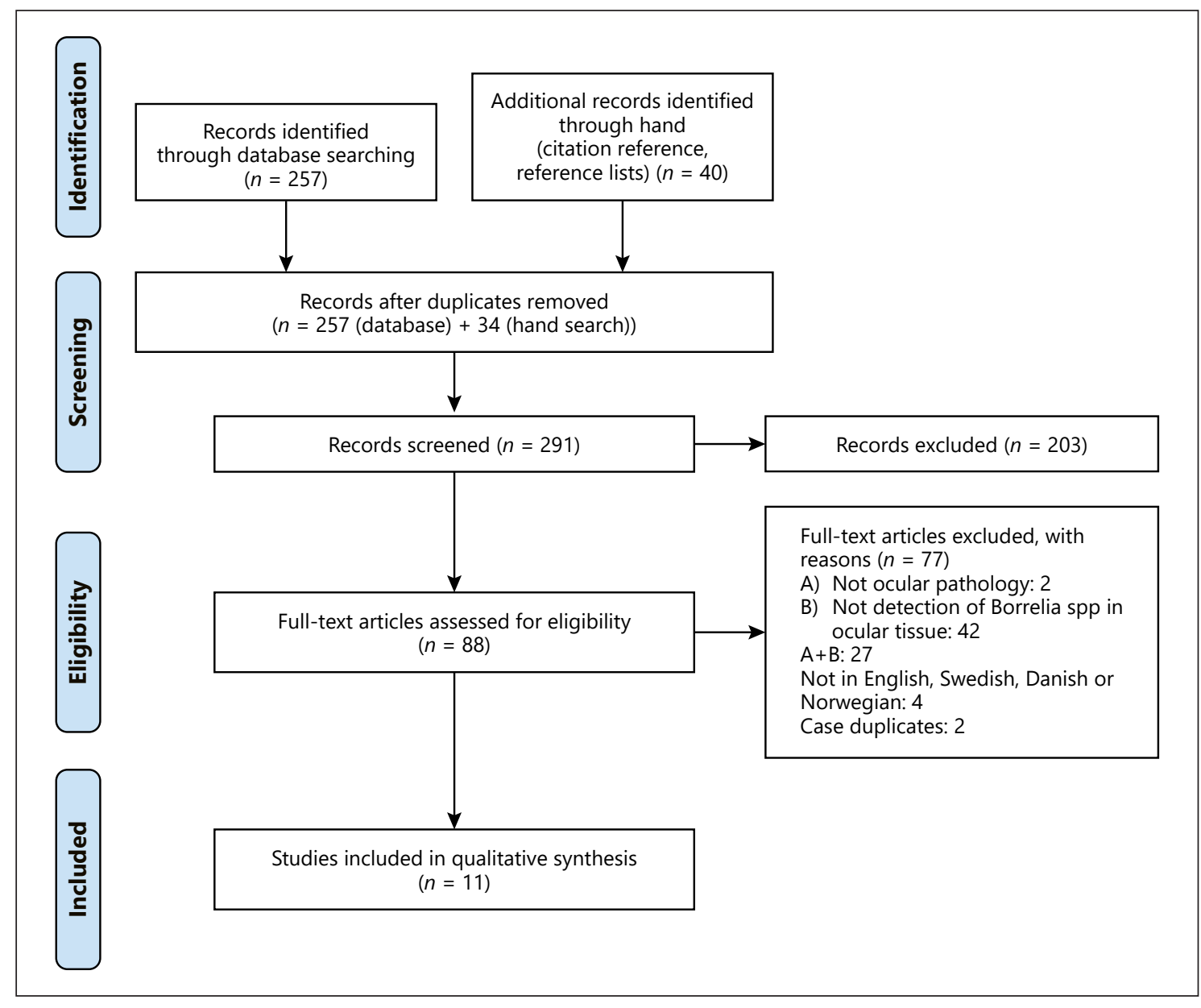

Fig. 1. Flow diagram of the study selection process.

were excluded; (B) detection of Borrelia spp. within the affected eye tissue; and (C) only studies written in English, Swedish, Danish, or Norwegian were included.

\section{Search Strategy}

In the scientific literature search, we included references comprising research on adults, young people, and children according to the predefined selection criteria. To construct the search string, the PubMed was first extensively searched in a nonsystematic manner to find articles fulfilling the inclusion criteria, either directly or through relevant references. This initial search yielded 12 articles that met the inclusion criteria. Subsequently, with the support of a medical librarian, a search string was constructed in a way that was assumed to capture all relevant studies and that also captured the 12 studies from the nonsystematic search: (lyme OR borrelia) AND (eye OR opht* OR blind* OR ocul*) AND (biopsy OR immunohisto* OR histology OR PCR OR polymerase OR tissue OR cytolog* OR microbiolog* OR microscop*) AND (humans). Two authors (B. Lindström and B. H. Skogman) independently systematically searched the PubMed for studies in January 2019 using the PubMed search string based on the eligibility criteria. Reference lists of articles that were considered relevant were searched. On the gathered studies fulfilling our inclusion criteria, a citation reference search was made through Google Scholar.

\section{Data Extraction and Quality Assessment}

The authors mentioned above independently reviewed the studies using a protocol (online suppl. material 1) to assess the clinical plausibility of Borrelia causing the eye condition. In case of disagreement, a third author (L. Tallstedt) made a final judgment. The quality of laboratory diagnosis in the reported cases was assessed with a protocol (online suppl. material 2) by the author K. Nilsson. As a secondary aim, the studies found with evidence for ocular Borrelia infection were evaluated for the possibility of good evidence-based treatment for ocular Borrelia infection. Disagreements between the reviewers were discussed and resolved through consensus. 


\section{Results}

\section{Search Results}

Our search strategy by PubMed on January 8, 2019, captured 257 studies with the search string (Fig. 1). Of these, 12 studies, found through direct hits, were assessed eligible and included for full-article review and quality assessment (Duray and Steere [4]; Kauffmann and Wormser [5]; Karma et al. [6]; Preac-Mursic et al. [7]; Clinch et al. [8]; Schubert et al. [9]; Karma et al. [10]; Hilton et al. [11]; Linna et al. [12]; Mikkila et al. [13]; Mikkila et al. [14]; and Dietrich et al. [2]). No additional studies were found through the reference lists. Citation references yielded one additional study, Hassan M. [15], that was included. Two out of the 13 primary studies included from the search were later excluded, Karma et al. [10] and Mikkila et al [13] since they contained duplicate cases from other articles by Karma et al. [6] and Mikkila et al. [14], see The Finnish cases below. Eleven studies were included as shown in Figure 1, of which all passed the clinical evaluation protocol. A new PubMed search with the same search string was conducted on first of April 2021, which yielded 31 new articles, none of which met the established inclusion criteria.

\section{Laboratory Diagnostic Categories}

It is claimed that in these cases Borrelia has been identified in the cornea, conjunctiva, the aqueous fluid, and the vitreous. The laboratory diagnostic characteristics of the included studies are presented in Table 1 and have been ranked into three categories: groups A, B, and C, based on their diagnostic quality. Group A represents substantial evidence of Borrelia detection, when the laboratory method corresponds to the genus level (Borrelia spp. or Borrelia burgdorferi sensu lato) or species level (e.g., B. afzelii, B. burgdorferi sensu stricto), together with a sufficiently detailed laboratory description to assume a correct performance. The studies marked in group B only have partial evidence for a strategy that admits the detection of Borrelia, but it is still plausible to assume that Borrelia was detected. They have a sufficient description of the laboratory technique, and the assumption of Borrelia infection is strengthened by either serology or EM, but the method used for direct detection of Borrelia cannot reach the level of genus or species. Those studies that did not fulfill the criteria, which correspond to group A or B, are marked as group $\mathrm{C}$ and are considered to have weak evidence for the detection of Borrelia infection.

\section{PCR as a Diagnostic Tool}

PCR has different diagnostic levels that correspond to the genus or species depending on the primers used. Dietrich et al. [2] claim they have identified the species Borrelia garinii with PCR. They used the flaB and ospA genes that might enable detection to species level. However, a fundamental requirement should be to present the primer target and the size of the amplified fragment, and this information is missing. Therefore, their diagnostic level is set at the genus level. None of the included cases reached the level of species.

\section{Microscopy as a Diagnostic Tool}

Four studies using microscopy had a sufficient description of their laboratory work; Duray and Steere [4]; Kauffmann and Wormser [5]; Linna et al. [12]; and Dietrich et al. [2]. Microscopy by itself cannot adequately sort out Borrelia from other potential spirochetes. We assume that microscopy without the help of other diagnostic methods can reach the family level, which Borrelia shares with the human pathogen Treponema. Treponema pallidum (syphilis) can cause similar manifestations to Borrelia as described by Karma and Mikkila [16]. In serology, the risk of cross-reactivity between Treponema and Borrelia is substantial; therefore, a positive Borrelia serology is always checked for Treponema, and hence, all cases with positive serologies can be counted on to have been tested negative for Treponema as shown in Table 1. A negative Borrelia serology is not tested for Treponema, but it is not likely to contain Treponema antibodies due to the cross-reactivity with Borrelia. Linna et al. [12] used tandem scanning confocal microscopy and Dietrich et al. [2] electron microscopy, both established methods which enable detailed examination. Duray and Steere [4] and Kauffmann and Wormser [5] used the common silver nitrate Dieterle staining method for light microscopy of spirochetes. Dietrich et al. [2] could achieve the genus level Borrelia through the additional use of PCR. Duray and Steere, Kauffman and Wormser, and Linna et al. $[4,5,12]$ had no supplementary method to microscopy, and they assessed to reach the level of the family Spirochaetaceae. Schubert et al. [9] used Papanicolaou staining which is not a fully established staining of spirochetes, and repeated serology for Borrelia was negative. Moreover, Shubert et al. [9] did not use defined Borrelia species as a control to verify the usefulness of their Papanicolaou staining, which is a mandatory requirement as they used a stain that is more appropriate for cytopathological assessment. They can therefore be assumed not to have used a correct laboratory method; they are assessed to reach the level of family Spirochaetaceae. 


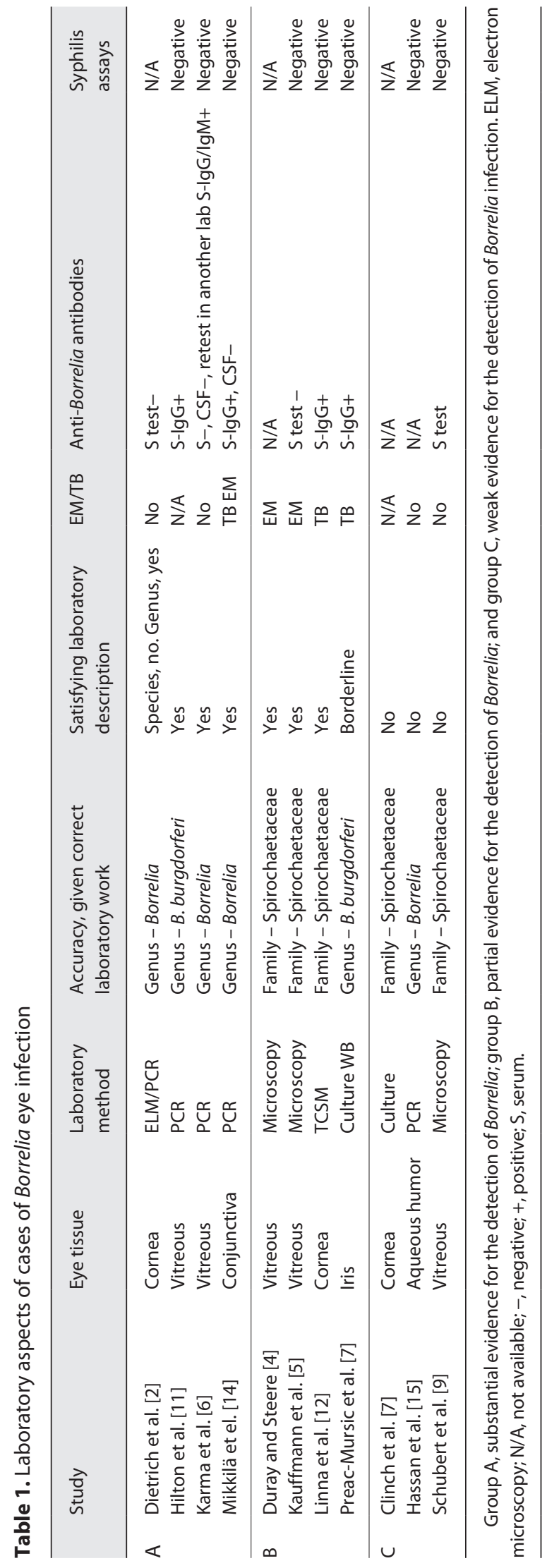

Borrelia in Ocular Tissue

\section{Culture as a Diagnostic Tool}

Two studies used culture, Preac-Mursic et al. [7] and Clinch et al. [8]. This is the only methodology that can prove a finding of viable Borrelia. Clinch et al. [8] have not reported how they determined their cultured organism to be Borrelia. We assume that they have used microscopy, which does not provide precision in species determination. Therefore, the diagnostic level is set at the family level, yet it is difficult to assess as the laboratory performance is insufficiently described. The case was assessed as having weak evidence for laboratory detection of Borrelia. Preac-Mursic et al. [7] have used monoclonal antibodies and Western blot (WB) techniques to demonstrate their cultured Borrelia. The diagnostic precision is estimated to be at the genus level. Preac-Mursic's description of the method of culture and WB are acceptable, but the presentation of the $\mathrm{WB}$ results is sparse, which was why the quality of the laboratory performance was evaluated as "borderline," and the laboratory evidence was assessed as partial.

\section{Clinical Diagnostic Aspects}

The clinical diagnostic aspects of the cases are summarized in Table 2. Hassan et al. [15] did not present clinical information about the case but did to some extent present the workup for differential diagnosis. Clinch et al. [8] and Hilton et al. [11] did not present any clinical information or information about differential diagnostics. Although it was difficult to determine the clinical probability of Borrelia infection in these three studies, we decided to include them for further evaluation. As presented in Table 2, in some of the cases, EM or a TB has not been reported. Some cases showed no, or few, extraocular signs or symptoms.

\section{Treatments Used}

The different types of treatment are summarized in Table 2. In the case reported by Kauffmann and Wormser [5], orbital cellulitis was suspected and nafcillin, gentamicin, and neomycin-polymyxin were administered as treatment. Although this treatment was probably not primarily aimed at a suspected Borrelia infection, we have chosen to present it in the table (Table 2). Three of the cases gave no information about treatment, and it concerns Duray and Steere [4], Clinch et al. [8], and Hassan [15]. Two of the cases, Mikkila et al. [14] and Dietrich et al. [2], seemed to have successfully treated all signs of ocular infection. They had both in common that intravenous ceftriaxone was used and that the infection was of the ocular surface, i.e., keratopathy and conjunctivitis. 
Table 2. Clinical aspects of cases of Borrelia eye infection

\begin{tabular}{|c|c|c|c|c|c|c|}
\hline & Study & Eye condition & $\begin{array}{l}\text { Other possibly related } \\
\text { manifestations }\end{array}$ & $\begin{array}{l}\text { Reported } \\
\text { TB }\end{array}$ & Antibiotics & $\begin{array}{l}\text { Immunosuppres- } \\
\text { sive therapy }\end{array}$ \\
\hline \multirow[t]{3}{*}{ A } & Dietrich et al. [2] & $\begin{array}{l}\text { Crystalline } \\
\text { keratopathy }\end{array}$ & Arthritis & No & $\begin{array}{l}\text { Ceftriaxone iv } 2 \mathrm{wk} \text { and topical } \\
\text { tetracycline } 2 \mathrm{yr}\end{array}$ & $\begin{array}{l}\text { Systemic mtx, cs, } \\
\text { and cs drops }\end{array}$ \\
\hline & Hilton et al. [11] & Pars planitis & $\mathrm{N} / \mathrm{A}$ & $\mathrm{N} / \mathrm{A}$ & $\begin{array}{l}\text { Ceftriaxone iv } 10 \mathrm{~d} \text { and po macrolide } \\
2 \mathrm{mo}\end{array}$ & N/A \\
\hline & Mikkilä et el. [14] & Conjunctivitis & $\begin{array}{l}\text { EM, fatigue, migratory arthralgia, } \\
\text { vertigo, tinnitus, and facial pain }\end{array}$ & Yes & $\begin{array}{l}\text { Repeated ceftriaxone iv } 2 \mathrm{wk} \text { and po } \\
\text { amoxicillin + probenecid po } 100 \mathrm{~d}\end{array}$ & No \\
\hline \multirow[t]{3}{*}{ B } & Duray and Steere [4] & Uveitis & EM & No & $\mathrm{N} / \mathrm{A}$ & N/A \\
\hline & Kauffmann et al. [5] & Panuveitis & $\begin{array}{l}\text { EM, headache, fever, chills, } \\
\text { nausea, and vomiting }\end{array}$ & No & $\begin{array}{l}\text { Cefazolin, nafcillin, and gentamycin iv; } \\
\text { chloramphenicol and gentamycin ivt; } \\
\text { topical neomycin-polymyxin B; and } \\
\text { sulphafurazole }\end{array}$ & cs iv and cs drops \\
\hline & Preac-Mursic et al. [7] & Panuveitis & No & Yes & Doxycycline po 4 wk, twice & $\begin{array}{l}\text { Yes. Not } \\
\text { specified }\end{array}$ \\
\hline \multirow[t]{3}{*}{ C } & Clinch et al. [7] & Keratitis & $\mathrm{N} / \mathrm{A}$ & N/A & N/A & N/A \\
\hline & Hassan et al. [15] & Iritis & $\mathrm{N} / \mathrm{A}$ & $\mathrm{N} / \mathrm{A}$ & $\mathrm{N} / \mathrm{A}$ & N/A \\
\hline & Schubert et al. [9] & Panuveitis & No & No & $\begin{array}{l}\text { Ceftriaxone iv } 3 \text { wk and doxycycline iv } \\
3 w k\end{array}$ & $\begin{array}{l}\text { Cs iv (+acyclovir } \\
\text { iv) }\end{array}$ \\
\hline
\end{tabular}

Group A, substantial evidence for the detection of Borrelia; group B, partial evidence for the detection of Borrelia; and group C, weak evidence for the detection of Borrelia infection. cs, corticosteroid; d, days; iv, intravenous; IVT, intravitreal treatment; mo, months; mtx, metothrexate; N/A, not available; po, peroral; wk, weeks; yr, years.

However, in both cases, other antibiotics had also been given, and the description of the final clinical result and the follow-up time could have been more precise. The other five cases had all unclear results of treatment. Treatment was often followed by improvement. None of the cases had complete resolution of possible infectious signs.

\section{The Finnish Cases}

Four studies, Karma et al. [6], Karma et al. [10], Mikkila et al. [13], and Mikkila et al. [14], published in the 1990s by a group of Finnish authors, met the inclusion criteria. Because of case duplicates, only two unique includible cases were found. One of the articles, from January 1999, described the laboratory work done in both cases [13]. One is a case of a 15-year-old girl with vitritis and retinitis pigmentosa-like retinal changes with positive PCR in the vitreous and CSF. This was first published in 1993 [6]. Later, that patient was also described as having neuroretinitis in an article published in 1995 [9], as well as in two later articles [13, 14]. To evaluate this case, we have used the original report [5] and added information about the laboratory work published in the article from January 1999 [13]. The other case is a woman with follicular conjunctivitis with positive PCR from the conjunctiva. That case was originally published in the study from January 1999 [13], describing the laboratory diagnosis. The complementary clinical description of the case was later published in July 2000 by Mikkila et al. [14].

\section{Studies in Other Languages}

Two articles in German were found but not analyzed in detail due to linguistic interpretation difficulties. These are Meier et al. [17] and Lohmann et al. [18]. Meier et al. [17] claimed that with microscopy that they had found $B$. burgdorferi in the vitreous of an eye with endophthalmitis. Lohman et al. [18] claimed PCR detection of B. burgdorferi in the corneas of two eyes with keratitis. 


\section{Discussion}

\section{Final Outcome of Eligible Cases}

This study was conducted to assess the evidence and state of the art that can be assumed to support an ocular infection of Borrelia spp. Apart from our case, we found 11 additional cases where authors claimed Borrelia to have been directly detected in relevant pathological eye tissue: Duray and Steere [4], Kauffmann and Wormser [5], Karma et al. [6], Preac-Mursic et al. [7], Clinch et al. [8], Schubert et al. [9], Hilton et al. [11], Linna et al. [12], Mikkila et al. [14], Dietrich et al. [2], and Hassan et al. [15]. In four of these 11 cases, we assessed them to have substantial laboratory evidence of Borrelia infection, and these are Karma et al. [6], Hilton et al. [11], Mikkila et al. [14], and Dietrich et al. [2]. In four additional cases, we assessed it was reasonable to suspect Borrelia infection: Duray and Steere [4], Kauffmann and Wormser [5], Preac-Mursic et al. [7], and Linna et al. [12] and for a further three: Clinch et al. [8], Schubert et al. [9], and Hassan [15]. Sufficient evidence to conclude a Borrelia infection as a cause was lacking. Two additional articles in German were found that were not included due to the language barrier. Consequently, these reports add more evidence to the concept of ocular Borrelia infection than stated by the French guidelines on Lyme Disease of 2019 by Gocko et al. [1].

\section{Assessment of the Usefulness of Laboratory Methods}

Microscopy without using PCR or direct immune detection of B. spirochetes has its limitations. Two other genera of spirochetes known as human pathogens are Leptospira and Treponema. Leptospira can usually be distinguished under the microscope through its specific morphology. Treponema species, causing the diseases syphilis, bejel, yaws, and pinta, shares the same family as Borrelia and is less easy to distinguish under the microscope. Many of the ophthalmological manifestations seen in Lyme disease have also been observed in syphilis [16]. It is therefore crucial to exclude laboratory evidence of syphilis when Borrelia is suspected using only microscopy. In theory, other spirochetes, perhaps not yet known as human pathogens, could be mistaken for Borrelia under the microscope. Also, if a correct staining for spirochetes is not performed, tissue debris can be mistaken for spirochetes [19]. The possibility of indirectly finding evidence for uveitis caused by Borrelia through CSF analysis of anti-Borrelia antibodies or PCR has been discussed [20,21]. In the case of simultaneous neurological symptoms, optic neuritis, or possibly neuroretinitis, such an approach might be feasible. If that is not the case, we believe that aspirations or biopsies of relevant ocular content are more accurate and offer better differential diagnostic opportunities.

Seronegative Borrelia infection is a known entity, most often associated with EM [22]. In the group A of cases with substantial evidence for detected Borrelia, the patient presented by Dietrich et al. [2] was found seronegative. In the Karma et al. [10] report, the patient was seronegative in the first test several months after contracting her eye disease, but a retest of the same sample in another laboratory showed positive s-IgM/IgG [10]. In the group $\mathrm{C}$ category with weak evidence for detected Borrelia, the patient described by Schubert et al. [9] was seronegative. Some of the cases had neither known TBs nor EM. This highlights that looking for Borrelia in ocular tissue samples can be relevant even if there is a lack of positive serology or other classical clues for borreliosis.

In this article, we have only discussed Borrelia infection of the eye. Also, an indirect affection to the eye by Borrelia is possible. Murillo et al. [23] described a case of probable immune-based keratopathy secondary to a likely Borrelia infection of the eyelid.

\section{Evaluation of Treatment Strategies}

Evaluation of treatment in the cases included in this review was not the primary aim. However, only two of the studies seemed to have noticed clear effects of treatment $[2,14]$, and in the rest of the studies included, treatment showed more ambiguous results. This does not mean the treatment in the other cases were without effect but that the case descriptions were too insufficient to draw clear conclusions. The common tendency for relapses after treatment of ocular borreliosis is described in the earlier literature [24]. Based on what emerges from this review of published cases of well-proven ocular Borrelia infection, larger studies are needed to determine which treatment for ocular Borrelia infection is optimal.

Lyme disease can sometimes be difficult to cure as persistent symptoms can be observed even after rigorous treatment and is called by some researchers as posttreatment Lyme disease syndrome. It is hypothesized that it could be due to the infection not being eradicated or a hyperresponsive immune reaction to antigenic debris of dead B. spirochetes [25-27].

There is, as yet, no established antibiotic treatment for intraocular Borrelia infection [28, 29]. To our knowledge, either oral doxycycline $[28,30]$ or daily intravenous antibiotics such as cephalosporins or penicillins for two to three weeks are currently the two main types of antibiotic treatments for ocular borreliosis. 
Doxycycline is effective in neuroborreliosis; however, it can be questionable in intraocular disease. To our knowledge, there are few studies of its intraocular penetration. With no references, the French guidelines for Lyme disease state that doxycycline should not be given in intraocular disease due to poor intraocular penetration [1]. Two studies of systemic doxycycline in horses [31, 32] and one study of systemic doxycycline in rabbits [33] showed poor intraocular concentrations of doxycycline. In our material, we had three cases that used oral doxycycline (Linna et al. [12], Preac-Mursic et al. [7], and our case). In none of the cases was the intraocular inflammation eradicated. Preac-Mursic et al. cultured viable Borrelia from the iris following two 4-week courses with oral doxycycline [7]. Thus, from what we have found in these case reports, the usefulness of doxycycline in intraocular Borrelia infections needs to be further studied. Intravenous treatment for 2 to 3 weeks has practical and economical inconveniences, also when considering the uncertain outcomes. Two of the cases in this review had to stop longterm intravenous cephalosporin treatment due to negative effects on hematopoiesis $[9,11]$. Biliary complications [34] and pseudomembranous colitis [35] from long-term treatment with ceftriaxone for Lyme disease are also described. In a review regarding the intraocular penetration of systemic antibiotics, meropenem achieved the bestdocumented therapeutics levels, and the cephalosporins ceftriaxone, cefazolin, and ceftazidime presented the 'second-best' evidence for intraocular penetration as opposed to cipro- and levofloxacin, aminopenicillin, piperacillin, and clarithromycin where available data did not support use in these situations [36]. Oral antibiotics would be a more convenient route of systemic antibiotics. Moxifloxacin and linezolid are both antibiotics with the best available evidence for intraocular penetration, better than cephalosporins [36]. Both also have good laboratory evidence for being effective against Borrelia [27]. In theory, oral treatment by moxifloxacin or linezolid is an interesting alternative for treating intraocular Borrelia. Also, trimethoprim shows some laboratory evidence indicating that it can be effective [36, 37]. Interestingly, disulfiram, the substance commonly known for the treatment of alcoholism, is under study as a potential future drug for longterm treatment of relapsing borreliosis [38].

Intravitreal antibiotic treatment is an interesting but seldom discussed alternative. Ampicillin, cefazolin, ceftazidime, clindamycin, erythromycin, moxifloxacin, oxacillin, and vancomycin are antibiotics that can be used for intravitreal treatment [39], and all of these have proved to be effective against Borrelia in vitro, of which cefazolin had the most favorable values of minimal inhibitory concentration and minimal bactericidal concentration [27]. When intravitreal antibiotics are used, one might consider giving simultaneous intravenous doses of the same antibiotic to maintain the antibiotic intraocular concentration for a longer period [39].

Jarish Herxheimer's reaction (JHR) is a bothersome inflammatory complication when killing spirochetes, which usually occurs within $24 \mathrm{~h}$. Administration of corticosteroids prior to antimicrobial treatment has been used to prevent development of severe JHR [40]. With the guidance of these studies, a model for treating ocular borreliosis might be intravitreal cefazolin, possibly with 1-2 doses of intravenous cefazolin the same day and simultaneously starting an oral course of moxifloxacin or linezolid. In the case of any intraocular JHR, it would be optimal to undertake a clinical control within $24 \mathrm{~h}$ and possibly to add a preventive dose of topical or systemic corticosteroids.

\section{Limitations of This Study}

In this review, we have focused on the B. spirochetes causing Lyme disease and not on other Borrelia species causing louse-borne relapsing fever and tick-borne relapsing fever, which are both diseases known to have ophthalmological manifestations [41, 42]. We have also not searched for possible eye manifestations caused by louseborne relapsing fever or tick-borne relapsing fever. Another limitation in our review is that we have only used the databases of PubMed and Google Scholar. We have focused on cases with evidence of laboratory detection of Borrelia in ocular tissues. Due to the low number of cases with this kind of evidence, it is pragmatic to also look at cases with indirect laboratory evidence but high clinical suspicion, as done by others [24]. There are cases described with high clinical and indirect laboratory suspicion of ocular Borrelia infection where ocular tissue samples have not detected Borrelia. A balanced clinical assessment must be given priority over individual test results [24]. Finally, it should also be remembered that evidence of Borrelia species in ocular tissue does not necessarily mean that Borrelia is the cause of the current ocular pathology.

\section{Conclusion}

This systematic review which focuses on the diagnosis of Borrelia infection in the eye, including all reported cases and our case report, supports evidence of ocular infection of Borrelia species. However, the amount of data are 
quite small, why the conclusions rely on detailed assessments of the individual reports. We can conclude that seronegative Borrelia infection occurs, why in these cases, looking for Borrelia in ocular tissue samples can be relevant. Further that microscopy without using PCR is not sufficient to confirm the diagnosis of Borrelia infection in ocular tissue. In the articles studied, there is also currently no clear evidence of a golden standard treatment for ocular Borrelia infection. However, the selection of articles was not focused on treatment why no definitive conclusion can be drawn, but from what emerged, oral doxycycline and amoxicillin were not always optimal drugs for the treatment of intraocular Borrelia infection. Further studies on treatment strategies are therefore warranted.

\section{Acknowledgments}

The authors thank the personnel of the medical library, Falu Hospital, Sweden.

\section{Statement of Ethics}

Written informed consent was obtained from the patient in the case report to publish details of the patient's medical case. For the rest of the study, an ethics statement is not applicable because this study is based exclusively on the published literature.

\section{Conflict of Interest Statement}

The authors have no conflicts of interest to declare.

\section{Funding Sources}

Funding was obtained from the Centre for Clinical Research Dalarna, Uppsala University (CKFUU-935990, CKFUU-939634).

\section{Author Contributions}

Björn E. Lindström contributed to study design, manuscript writing - original draft, and evaluation of cases. Barbro H. Skogman contributed to study design, manuscript writing - review and editing, and evaluation of cases. Annika K. Lindström contributed to study design and manuscript writing - review and editing. Leif Tallstedt and Kenneth Nilsson contributed to study design, manuscript writing - review and editing, and evaluation of cases. All the authors approved the submitted version of the manuscript.

\section{Data Availability Statement}

The evaluation sheets of the review are added as online supplementary material.

\section{References}

1 Gocko X, Lenormand C, Lemogne C, Bouiller $\mathrm{K}$, Gehanno JF, Rabaud C, et al. Lyme borreliosis and other tick-borne diseases. Guidelines from the French scientific societies. Med Mal Infect. 2019 Aug;49(7):296-317.

2 Dietrich T, Geissdörfer W, Schlötzer-Schrehardt U, Holbach L, Schoerner C, Seitz B. Borrelia-associated crystalline keratopathy with intracorneal detection of Borrelia garinii by electron microscopy and polymerase chain reaction. Cornea. 2008 May;27(4):498-500.

3 Moher D, Shamseer L, Clarke M, Ghersi D, Liberati A, Petticrew M, et al. Preferred reporting items for systematic reviews and meta-analyses protocols (PRISMA-P) 2015 statement. Syst Rev. 2015 Jan 1;4(1):1.

4 Duray PH, Steere AC. The spectrum of organ and systems pathology in human Lyme disease. Zentralbl Bakteriol Mikrobiol Hyg A. 1986 Dec;263(1-2):169-78.

5 Kauffmann DJ, Wormser GP. Ocular Lyme disease: case report and review of the literature. Br J Ophthalmol. 1990 Jun;74(6):325-7.

6 Karma A, Pirttilä TA, Viljanen MK, Lähde YE, Raitta CM. Secondary retinitis pigmentosa and cerebral demyelination in Lyme borreliosis. Br J Ophthalmol. 1993 Feb;77(2):120-2.
7 Preac-Mursic V, Pfister HW, Spiegel H, Burk R, Wilske B, Reinhardt S, et al. First isolation of Borrelia burgdorferi from an iris biopsy. J Clin Neuroophthalmol. 1993 Sep;13(3):15561; discussion 162.

8 Clinch TE, Palmon FE, Robinson MJ, Cohen EJ, Barron BA, Laibson PR. Microbial keratitis in children. Am J Ophthalmol. 1994 Jan; 117(1):65-71.

9 Schubert HD, E Greenebaum E, Neu HC. Cytologically proven seronegative Lyme choroiditis and vitritis. Retina. 1994;14(1):39-42.

10 Karma A, Seppälä I, Mikkilä H, Kaakkola S, Viljanen M, Tarkkanen A. Diagnosis and clinical characteristics of ocular Lyme borreliosis. Am J Ophthalmol. 1995 Feb;119(2):12735.

11 Hilton E, Smith C, Sood S. Ocular Lyme borreliosis diagnosed by polymerase chain reaction on vitreous fluid. Ann Intern Med. 1996 Sep;125(5):424-5.

12 Linna T, H Mikkila H, Karma A, Seppala I, Petroll WM, Tervo T. In vivo confocal microscopy: a new possibility to confirm the diagnosis of Borrelia keratitis? Cornea. 1996 Nov;15(6):639-40.
13 Mikkila H, Karma AA, Viljanen MM, I Seppala I. The laboratory diagnosis of ocular Lyme borreliosis. Graefes Arch Clin Exp Ophthalmol. 1999 Mar;237(3):225-30.

14 Mikkila HO, Seppala IJ, Viljanen MK, Peltomaa MP, Karma A. The expanding clinical spectrum of ocular lyme borreliosis. Ophthalmology. 2000 Mar;107(3):581-7.

15 Hassan M, Sadiq MA, Agarwal A, Bodaghi B, Nguyen QD. Anterior segment manifestations of Lyme disease. In: Gupta V, Nguyen Q, LeHoang P, Agarwal A, editors. The Uveitis Atlas. New Delhi: Springer; 2020.

16 Karma A, Mikkilä H. Ocular manifestations and treatment of Lyme disease. Curr Opin Ophthalmol. 1996 Jun;7(3):7-12.

17 Meier P, Blatz R, Gau M, Spencker FB, Wiedemann P. [Pars plana vitrectomy in Borrelia burgdorferi endophthalmitis]. Klin Monbl Augenheilkd. 1998 Dec;213(6):351-4.

18 Lohmann CP, Winkler von Mohrenfels C, Gabler B, Reischl U, Kochanowski B. Polymerase chain reaction (PCR) for microbiological diagnosis in refractory infectious keratitis: a clinical study in 16 patients. Klin Monbl Augenheilkd. 2000 Jul;217(1):37-42. 
19 Murray PR, Rosenthal KS, Pfaller MA. Medical microbiology. 5th ed. USA: Elsevier Mosby; 2005. p. 430. Isbn 0-323-03303-2.

20 Wallet F, P Labalette P, Herwegh SS, C Loiez C, F Margaron F, R Courcol R. Molecular diagnosis of a bilateral panuveitis due to Borrelia burgdorferi sensu lato by cerebral spinal fluid analysis. Jpn J Infect Dis. 2008 May; 61(3):214-5.

21 Mahne J, Kranjc BS, Strle F, Ružić-Sabliić E, Arnež M. Panuveitis caused by Borrelia burgdorferi sensu lato infection. Pediatr Infect Dis J. 2015 Jan;34(1):102-4.

22 Oksi J, Uksila J, Marjamäki M, Nikoskelainen J, Viljanen MK. Antibodies against whole sonicated Borrelia burgdorferi spirochetes, 41-kilodalton flagellin, and $\mathrm{P} 39$ protein in patients with PCR- or culture-proven late Lyme borreliosis. J Clin Microbiol. 1995 Sep;33(9): 2260-4.

23 Murillo G, Ramírez B, Romo LA, MuñozSanz A, Hileeto D, Calonge M. Oculopalpebral borreliosis as an unusual manifestation of Lyme disease. Cornea. 2013 Jan;32(1):8790.

24 Bernard A, Seve P, Abukhashabh A, RoureSobas C, Boibieux A, Denis P, et al. Lyme-associated uveitis: clinical spectrum and review of literature. Eur J Ophthalmol. 2020 Sep; 30(5):874-85.

25 Bockenstedt LK, Gonzalez DG, Haberman AM, Belperron AA. Spirochete antigens persist near cartilage after murine Lyme borreliosis therapy. J Clin Invest. 2012 Jul;122(7): 2652-60.

26 Feng J, Wang T, Shi W, Zhang S, Sullivan D, Auwaerter PG, et al. Identification of novel activity against Borrelia burgdorferi persisters using an FDA approved drug library. Emerg Microbes Infect. 2014 Jul;3(7):e49.
27 Pothineni VR, Wagh D, Babar MM, Inayathullah M, Solow-Cordero D, Kim KM, et al. Identification of new drug candidates against Borrelia burgdorferi using high-throughput screening. Drug Des Devel Ther. 2016;10: 1307-22.

28 Weinberg RS. Ocular Involvement in Lyme Disease. American Academy of Ophthalmology. 2008. Available from: https://www.aao. org/current-insight/ocular-involvement-inlyme-disease.

29 Meyerhoff JO. Lyme disease treatment \& management. Medscape, Drug \& Diseases. 2019. Available from: https://emedicine.medscape.com/article/330178-treatment\#d11.

30 Feldman BH, Rodriguez-Garcia A, Lozano AFI, Palestine A, Altufeile KA, Koretz Z, et al. Lyme Disease. American Academy of Ophthalmology, EyeWiki. 2020. Available from: https: //eyewiki.org/Lyme_Disease\#General_ treatment.

31 Gilmour MA, Clarke CR, Macallister CG Dedeo JM, Caudell DL, Morton RJ, et al. Ocular penetration of oral doxycycline in the horse. Vet Ophthalmol. 2005 Sep-Oct;8(5): 331-5.

32 Davis JL, Salmon JH, Papich MG. Pharmacokinetics and tissue distribution of doxycycline after oral administration of single and multiple doses in horses. Am J Vet Res. 2006 Feb; 67(2):310-6.

33 Salminen L. Penetration of ocular compartments by tetracyclines. II. An experimental study with doxycycline. Graefes Arch Clin Exp Ophthalmol. 1977 Nov;204(3):201-7.

34 Ettestad PJ, Campbell GL, Welbel SF, Genese CA, Spitalny KC, Marchetti CM, et al. Biliary complications in the treatment of unsubstantiated Lyme disease. J Infect Dis. 1995 Feb; 171(2):356-61.
35 Puri BK, Hakkarainen-Smith JS, Monro JA The potential use of cholestyramine to reduce the risk of developing Clostridium difficileassociated diarrhoea in patients receiving long-term intravenous ceftriaxone. Med Hypotheses. 2015 Jan;84(1):78-80.

36 Brockhaus L, Goldblum D, Eggenschwiler L, Zimmerli S, Marzolini C. Revisiting systemic treatment of bacterial endophthalmitis: a review of intravitreal penetration of systemic antibiotics. Clin Microbiol Infect. 2019 Nov; 25(11):1364-9.

37 Reisinger EC, Wendelin I, Gasser R. In vitro activity of trimethoprim against Borrelia burgdorferi. Eur J Clin Microbiol Infect Dis. 1997 Jun;16(6):458-60.

38 Kaul L, Süss R, Zannettino A, Richter K. The revival of dithiocarbamates: from pesticides to innovative medical treatments. iScience. 2021 Jan;24(2):102092.

39 Barry P, Cordoves L, Gardner S. ESCRS Guidelines for Prevention and Treatment of Endophthalmitis Following Cataract Surgery: Data, Dilemmas and Conclusions. Co Dublin, Ireland: European Society of Cataract and Refractive Surgeons; 2013. Available from: https: //www.escrs.org/downloads/endophthalmitis-guidelines.pdf.

40 Belum RB, Belum WR, Arudra SKC, Reddy BSN. The Jarish-Herxheimer reaction: Revisited. Trav Med Inf Dis. 2013 Jul-Aug;11(4): 231-7.

41 European Centre for Diseases Prevention and Control. Facts about louse-borne relapsing fever. ECDC; 2015. Available from: https:// www.ecdc.europa.eu/en/louse-borne-relapsing-fever/facts.

42 European Centre for Diseases Prevention and Control. Facts about tickborne relapsing fever. ECDC; 2016. Available from: https:// www.ecdc.europa.eu/en/tickborne-relapsing-fever/facts. 